\title{
Physical exercise for strengthening innate immunity during COVID-19 pandemic: 4th series of scientific evidence
}

\author{
Yong-Seok Jee (iD https://orcid.org/0000-0001-6797-0843
}

Exercise refers to a series of processes that can improve physical fitness and overall health, including a heightened sense of well-being, through physical activity. Gaining these beneficial effects is possible through regular exercise that requires at least $30 \mathrm{~min}$ a day, 3 days a week, for 6 months. Regular exercise strengthens the body's organs, thereby providing protection from disease or aiding in any recovery processes. More specifically, regular exercise plays an important role in protecting the body from external antigens by enhancing immune function. The recent coronavirus disease 2019 (COVID-19) pandemic has caused much suffering and death to millions of people around the world. Although many young and healthy people recover from the coronavirus, it can be fatal to people with poor health or other illnesses. On the other hand, the immune system, which is the body's defense against illnesses, can be developed so that the body can recover from infectious diseases. This can be seen from cases where athletes infected with the coronavirus returns to the game or competition due to the production of antibodies.

The immune system refers to cells, tissues, and organs that are involved in the immune response in the body. It is the body's defense system that recognizes foreign substances that have entered the body as antigens and produces antibodies to fight against them. The purpose of this fourth editorial is to present my views on the relationship between regular exercise and the immune system, and to specifically discuss the role of exercise in relation to the body's innate immunity.

Everyone is born with innate immunity, which provides general protection from pathogens. Innate immunity fights against certain viruses that cause human illness and disease. The innate, nonspecific immune system is actually composed of cellular and physical defenses that do not rely on prior exposure to specific pathogens. The cellular components of the innate immune system include natural killer (NK) cells, phagocytes, neutrophils, and macrophages. The physical barrier includes the skin and mucous membranes associated with the lymphatic tissue and is located in areas where pathogens invade, such as the airways, gastrointestinal tract, and urogenital tract. The reticuloendothelial system is mainly composed of phagocytes, which are important for eliminating bloodrelated infections. Macrophages secrete cytokines that participate in phagocytosis, kills cells through the secretion of toxic chemicals, improves antigenic processes, reduces inflammation, activates helper $\mathrm{T}$ cells, and increases systemic responses to infection or damage. NK cells directly bind to and kill viral infectious cells and cancer cells. It also functions as a killer cell in antibody-dependent cytotoxicity.

To date, many studies have reported on leukocytes, including subtypes and NK cells among innate immune cells related to exercise. These changes in immune cells through exercise have been studied as follows. First, the effects of exercise related to the function of innate immune cells is a field in which comparisons are made between the innate immune cells of trained athletes and sedentary people. Results generally point to a higher immune function in athletes versus non-physically active people. Second, it is a field related to investigating the changes in immune cell func- 
tions in subjects who train for a certain period of time with various intensities and durations under specific conditions. It is known that the number of leukocytes (leukocytes, neutrophils, lymphocytes) and NK cells in exercise groups are higher than control groups. Moreover, it is known that leukocytes and NK cells increase after moderate exercise, as well as after incremental maximal exercise. In addition, when the intensity of exercise is gradually increased and the maximum level is reached, leukocytes, neutrophils, lymphocytes, T cells, B cells, and NK cells increase, while the T4/T8 ratio decreases. NK cells and T8 cells have a greater density of $\beta_{2}$-adrenergic receptors than T4 cells, which may explain why these cells increase so significantly after moderate or maximal exercise. As such, an increase in the number and function of NK cells during exercise provide additional defense capabilities and is thought to compensate for immune vulnerability due to a temporary decrease in the $\mathrm{T} 4 / \mathrm{T} 8$ ratio.

NK cells are unique in expressing spontaneous cytolytic activity against various tumor and virus-infected cells as part of the innate immune system. Unlike T lymphocytes, NK cells do not require the participation of antibodies or major histologic synthetic antigens for initial toxicity, and react rapidly to foreign substances. When the antigen-specific immune system starts to respond, they are controlled by NK cells, which account for about $15 \%$ of lymphocytes and is thought to play an important role in the first line of defense against the spread of malignant diseases. Therefore, it is important to elucidate the effects of exercise on NK cell numbers and activity. In particular, greater attention should be given to the role of exercise and how innate immunity system can be strengthened, especially at the present time when the coronavirus is a grave threat to the well-being and lives of people everywhere.

So, the question is, how much exercise is required to improve our innate immunity, thereby improving our defense against contracting the coronavirus? For patients who have already contracted the coronavirus, how much exercise will be required to help them recover, and what percentage of the immune cells will be positively changed? I believe that addressing these questions will help contribute to the global fight against the current COVID-19 pandemic.

The first series is presented in J Exerc Rehabil 2019;15(3):339340, Available from: https://doi.org/10.12965/jer.1938302.151

The second series is presented in J Exerc Rehabil 2020;16(2): 113-114, Available from: https://doi.org/10.12965/jer.2040178. 089

The third series is presented in J Exerc Rehabil 2020;16(3): 205-206, Available from: https://doi.org/10.12965/jer.2040414. 207

\section{CONFLICT OF INTEREST}

No potential conflict of interest relevant to this article was reported.
Research Institute of Sports and Industry Science, Hanseo University 46 Hanseo 1-ro, Haemimyeon, Seosan 31962, Korea E-mail: jeeys@hanseo.ac.kr 